\title{
Efectos del cruzamiento de ovejas Ideal con carneros Merino Multipropósito sobre la morfología de piel y producción de lana
}

\author{
Flores Quintana, C. ${ }^{1}$; Yáñez, E.A. ${ }^{2}$; Carlino, M.N. ${ }^{3}$
}

${ }^{1}$ Departamento Ciencias Básicas, ${ }^{2}$ Departamento Producción Animal, ${ }^{3}$ actividad privada, Facultad de Ciencias Veterinarias, Universidad Nacional del Nordeste, Sargento Cabral 2139, Corrientes (3400), Tel/Fax. 0379-4425753.

E-mail: carolina@vet.unne.edu.ar.

\begin{abstract}
Resumen
Flores Quintana, C.; Yáñez, E.A.; Carlino, M.N.: Efectos del cruzamiento de ovejas Ideal con carneros Merino Multipropósito sobre la morfología de piel y producción de lana. Rev. vet. 24: 1, 14-20, 2013. En Argentina la producción de lanas finas es insignificante y esto representa una limitante para el crecimiento lanero del país, pues la tendencia del mercado mundial muestra su preferencia por este tipo de lanas. En la Provincia de Corrientes se presenta la misma problemática y como alternativa para aumentar la producción de lanas finas sin disminuir la producción de carne ovina se introdujeron ejemplares de Merino Multipropósito (MPM), persistiendo la necesidad de conocer cómo repercute sobre los demás parámetros productivos y cuál es la base estructural que establece este mejoramiento en la calidad de lana. El objetivo del trabajo fue evaluar el efecto del cruzamiento de ovejas Ideal con machos MPM, sobre las características de la piel y la producción y calidad de lana. Los parámetros evaluados fueron diámetro y longitud de fibra, peso y rendimiento de vellón, densidad de folículos y relación de folículos secundarios y primarios $(\mathrm{S} / \mathrm{P})$. Los parámetros productivos se determinaron con el protocolo INTA PROVINO. Para el recuento de folículos primarios y secundarios se realizaron biopsias de piel. Las características de producción y calidad de lana se consideraron entre borregas contemporáneas. Las ovejas Ideal, borregas Ideal y borregas F1 Ideal x MPM presentaron diámetro de fibra de 24,30; 22,24 y 21,06 $\mu \mathrm{m}$, respectivamente. $\mathrm{F} 1$ presentó diámetros significativamente menores. Los otros parámetros productivos evaluados no presentaron diferencias estadísticas. La relación S/P fue mayor en las borregas F1 (Ideal x MPM) en relación con Ideal, en tanto que la densidad de folículos no mostró diferencias entre los animales contemporáneos. La comparación realizada en este trabajo permitió observar una disminución del diámetro de fibra en borregas F1 (Ideal $\mathrm{x}$ MPM), que puede explicarse por la mayor relación S/P, sin disminución del peso del vellón.
\end{abstract}

Palabras clave: ovino, histología de piel, densidad folicular, relación folículos secundarios/ primarios.

\begin{abstract}
Flores Quintana, C.; Yáñez, E.A.; Carlino, M.N.: Crossbreeding effects of Multipurpose Merino rams with Ideal ewes on skin morphology and wool production. Rev. vet. 24: 1, 1420, 2013. In Argentina, fine wool production is insignificant and this represents a limitation to wool industry of the country, as the global market tendency shows a preference for this type of raw material. Corrientes Province, in Argentina, has the same problem. An alternative to increase fine wool production without reducing meat production was the introduction of the Multipurpose Merino (MPM) which led to the need of understanding the impact on productive parameters and the structural basis that determines the improvement on wool quality. The objective was to evaluate the effect of crossing Ideal sheep with MPM rams, on the skin characteristics, wool production and quality. Studied parameters were fiber diameter, fiber length, weight, fleece performance, follicle density and secondary/primary follicles ratio (S/P). Performance parameters were determined with INTA PROVINO protocol. Skin biopsies were performed for counting primary and secondary follicles of skin. Production and quality characteristics of wool were considered on same age ewes. Ideal sheep, Ideal ewe lamb and F1 Ideal x MPM showed fiber diameters of 24.30, 22.24 and $21.06 \mu \mathrm{m}$ respectively, with significantly lower diameter at F1 crossbred. The other production parameters did not differ. The $\mathrm{S} / \mathrm{P}$ ratio was greater in $\mathrm{F} 1$ ewes in relation to the Ideal, while the density of follicles showed no differences between contemporary animals. The comparison made in this
\end{abstract}


work allowed us to observe a decrease in fiber diameter in sheep F1 Ideal x MPM, which can be explained by the higher $\mathrm{S} / \mathrm{P}$ ratio, without reduction of fleece weight.

Key words: sheep, skin histology, follicular density, secondary/primary follicular ratio.

\section{INTRODUCCIÓN}

La lana es una de las fibras textiles más utilizadas desde tiempos remotos; la producción mundial fue de 1.992.000 t en el año 2010. Australia, Nueva Zelanda, Argentina y Uruguay son los productores líderes. En Argentina la producción de la zafra 2011/12 fue de $44.000 \mathrm{t}$, de las cuales fueron exportadas $40.264 \mathrm{t}$ por un valor de u $\$ \mathrm{~s} 225.000{ }^{9}$. Durante los últimos 20 años ocurrieron marcados cambios en la cadena lanera textil, con concentración de la industria en China y desaparición del procesamiento de la lana en Australia. Sudamérica, liderada por Uruguay y Argentina, constituye el segundo polo a nivel mundial de procesamiento primario de la lana.

Los cambios mencionados generan un nuevo escenario mundial en el cual se desarrollará la actividad ovina. Países que tradicionalmente criaban razas de doble propósito sobre la base Corriedale, como Uruguay, Sur de Brasil, Argentina, Chile y Perú, cambiarán hacia un doble propósito con lanas más finas dentro del Corriedale, o mediante el cruzamiento con otros genotipos que afinen su lana y mantengan o mejoren la producción de carne (Merino, Dohne Merino, Merino Multipropósito MPM).

El $61,9 \%$ de la producción de lana argentina proviene de la región patagónica ${ }^{9}$. En las provincias de Chubut y Río Negro se trabaja principalmente con la raza Merino, productora de las lanas más finas, cuyo grosor oscila entre 13 y $24 \mu \mathrm{m}$. Además existen otras dos áreas diferenciadas: el Litoral con las razas Corriedale, Ideal y Romney, con producción de lanas cruza fina y cruza gruesa, y el Noroeste poblado por razas criollas, con su producción destinada a tejidos artesanales.

La lana fue perdiendo participación en el mercado mundial de fibras, desde un $10 \%$ en 1960 al actual 2,4\% en 2011, al competir con los tejidos sintéticos y el algodón, ya que las relaciones de precios actuales equivalen aproximadamente a 3:1 o 4:1. Para lograr que la lana obtenida posea mayor valor y demanda, es necesario trabajar sobre el afinamiento de la fibra, sin descuidar la cantidad de lana producida y las tasas de procreo de la majada. La disminución del diámetro de la fibra depende de la cantidad de folículos secundarios presentes en la piel y de la presencia de folículos primarios más pequeños. Para incrementar la densidad folicular y el peso del vellón, es necesario reducir el tamaño de los folículos primarios y aumentar la ramificación de los folículos secundarios. Tanto la densidad como la longitud de la fibra estarían controlados por genes que regulan el número, distribución e intensidad de la señal de las células pre-papilares que se originan en la piel del feto, a partir de los fibroblastos de la dermis.

El Merino Multipropósito (MPM) es una innovación introducida desde Australia a Sudamérica, con el objeto de lograr lanas superfinas especiales, manteniendo alta tasa reproductiva, velocidad de crecimiento y carcasas magras. Las lanas especiales obtenidas estarían determinadas por una reducción en el diámetro de los folículos primarios a una finura equivalente o menor que la de los secundarios, aumentando notablemente la densidad de folículos en la piel. Los animales criados bajo el sistema de selección SoftRollingSkin $(\mathrm{SRS} \AA)$, origen del MPM, tendrían poblaciones de células pre-papilares más numerosas que ovinos de otros grupos genéticos ${ }^{10,11}$.

A nivel nacional no se encuentran trabajos publicados que investiguen la densidad (número de folículos/ $\mathrm{mm}^{2}$ ) y el arreglo folicular en las razas ovinas puras y en sus cruzas. La importancia de estudiar la densidad folicular, la relación entre folículos secundarios y primarios (S/P) y el arreglo de los folículos pilosos a nivel de piel, radica en que permite cuantificar los avances alcanzados con los cruzamientos utilizados, detectando aquellos animales que tienen baja relación $\mathrm{S} / \mathrm{P}$ y reducido número de folículos secundarios derivados. Existe un hecho que no ha sido demostrado consistentemente, en el sentido que al lograr lanas de mayor calidad y valor comercial, la cantidad de lana producida sería menor. Sin embargo, si se considera que la reducción en el diámetro de la lana en los ovinos MPM se produce por aumento de la densidad de folículos y de la relación $\mathrm{S} / \mathrm{P}$, el peso del vellón no debería modificarse, manteniéndose el volumen producido y aumentando su valor comercial.

El indicador $\mathrm{W} / \mathrm{D}$, que relaciona el peso del vellón limpio y el diámetro de la fibra de lana ${ }^{2}$, podría utilizarse como un indicador del impacto económico del MPM sobre el sistema de producción de lana, relacionándolo con el valor de mercado de la lana del micronaje obtenido .

El objetivo del presente trabajo fue evaluar en la progenie F1 el impacto del cruzamiento de ovejas de raza Ideal con machos MPM, sobre características de la piel (densidad folicular y relación $\mathrm{S} / \mathrm{P}$ ) y características de la lana (longitud y diámetro de las fibras, peso del vellón sucio y rendimiento al lavado).

\section{MATERIAL Y MÉTODOS}

El trabajo se realizó sobre majadas comerciales del Departamento Monte Caseros (Corrientes, Argentina). Se evaluaron las modificaciones de las características 
de piel comparando madres de raza Ideal y la primera generación descendiente del cruzamiento Ideal x MPM. Los parámetros evaluados fueron diámetro de fibra, densidad de folículos y relación S/P. Para el recuento de folículos primarios y secundarios se realizaron biopsias de piel con auxilio de un "sacabocado" de 1 $\mathrm{cm}$ de diámetro (Figura 1.A). La zona elegida se situó $10 \mathrm{~cm}$ atrás de la paleta y de la línea media. La piel se higienizó con alcohol 70\% y la extracción se efectuó bajo anestesia local (lidocaína al 2\%). La piel extraída fue fijada con formol tamponado al $10 \%$ por espacio de 7 días. Posteriormente se procesó según la técnica de McCloghry ${ }^{21}$.

Los cortes de $8 \mu \mathrm{m}$ fueron paralelos a la superficie de la piel; las técnicas de coloración utilizada fueron hematoxilina-eosina (HyE) y Sapicsatin (Figura 2. A y B). La cuantificación de los folículos se realizó con imágenes obtenidas con aumento de 10X, usando el software Image-ProPlus, versión 5.1, Media Cybernetics Inc., evaluándose entre 5,15 y $15,88 \mathrm{~mm}^{2}$ de piel por animal. Para determinar la densidad, se contaron los folículos de 5 campos (de 0,94 x 1,24 mm cada uno), tomados al azar en zonas donde el corte era completo y sin estiramiento. En los márgenes se consideraron solo aquellos folículos situados en el borde superior e izquierdo, para no sobrestimar su número.

Para obtener la relación S/P se identificaron y demarcaron los folículos primarios, que fueron recono-
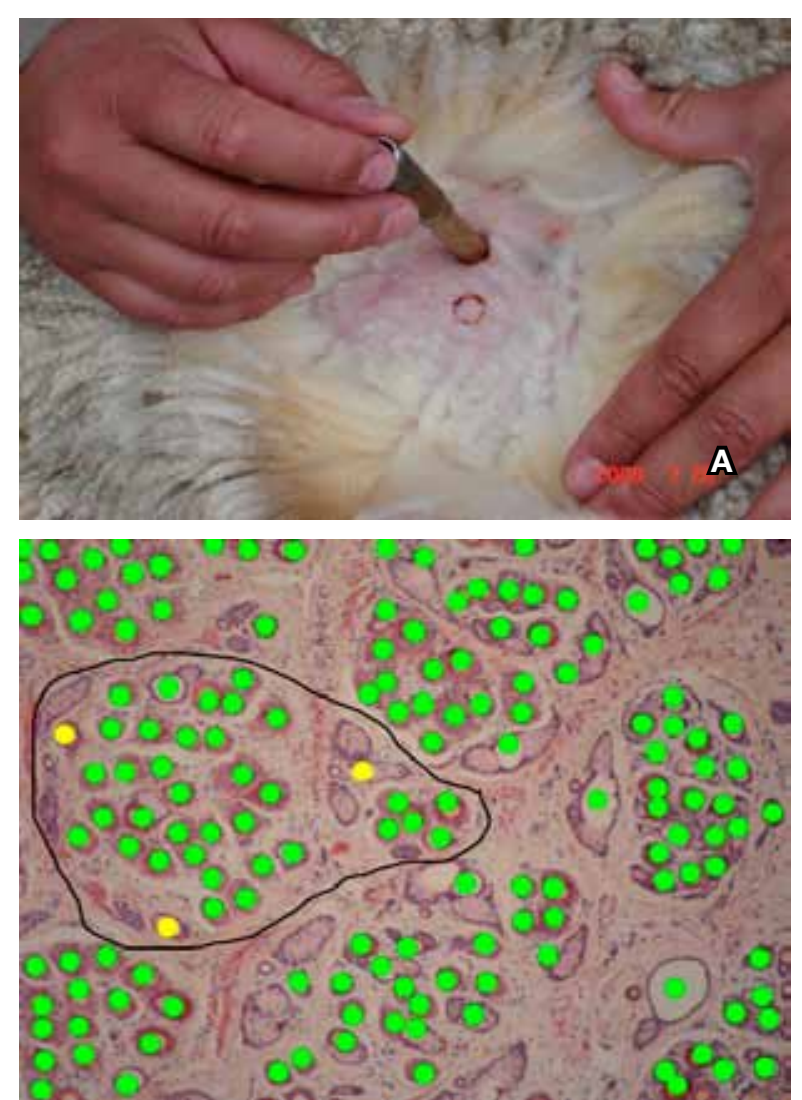

Figura 1. A: obtención de la muestra de piel con auxilio del sacabocado. B: corte histológico de piel ovina (10X), mostrando la marcación de folículos primarios (amarillos) y secundarios (verdes). cidos través de estructuras accesorias como glándula sudorípara, glándula sebácea bilobulada, músculo piloerector y por la posición del grupo folicular. Los restantes se caracterizaron como folículos secundarios (Figura 1.B). Posteriormente se compararon las características productivas y la calidad de lana entre borregas de raza Ideal puras y las descendientes del cruzamiento de madres Ideal con machos MPM (borregas F1). Los parámetros evaluados fueron el peso del vellón sucio y limpio, la longitud de mecha, el rendimiento al lavado, el diámetro de la fibra y el índice W/ $\mathrm{D}^{2}$.

El peso de vellón sucio se determinó con balanza electrónica al momento de la esquila. La longitud de mecha se obtuvo midiendo con regla la distancia entre la base y la punta de la fibra. El rendimiento al lavado y el diámetro de fibra fueron parámetros determinados en el Laboratorio de Lanas del INTA Bariloche, a partir de la muestra obtenida en el flanco del animal, entre la segunda y tercera costillas, bajo protocolo del INTA para PROVINO. La determinación del parámetro W/D se realizó calculando la relación entre el peso de vellón limpio dividido por el cubo del diámetro de fibra.

Para cada tratamiento se utilizaron 20 animales mantenidos en el mismo potrero y bajo las mismas condiciones de manejo, sanidad y alimentación. Las madres fueron ovejas adultas que se seleccionaron en-
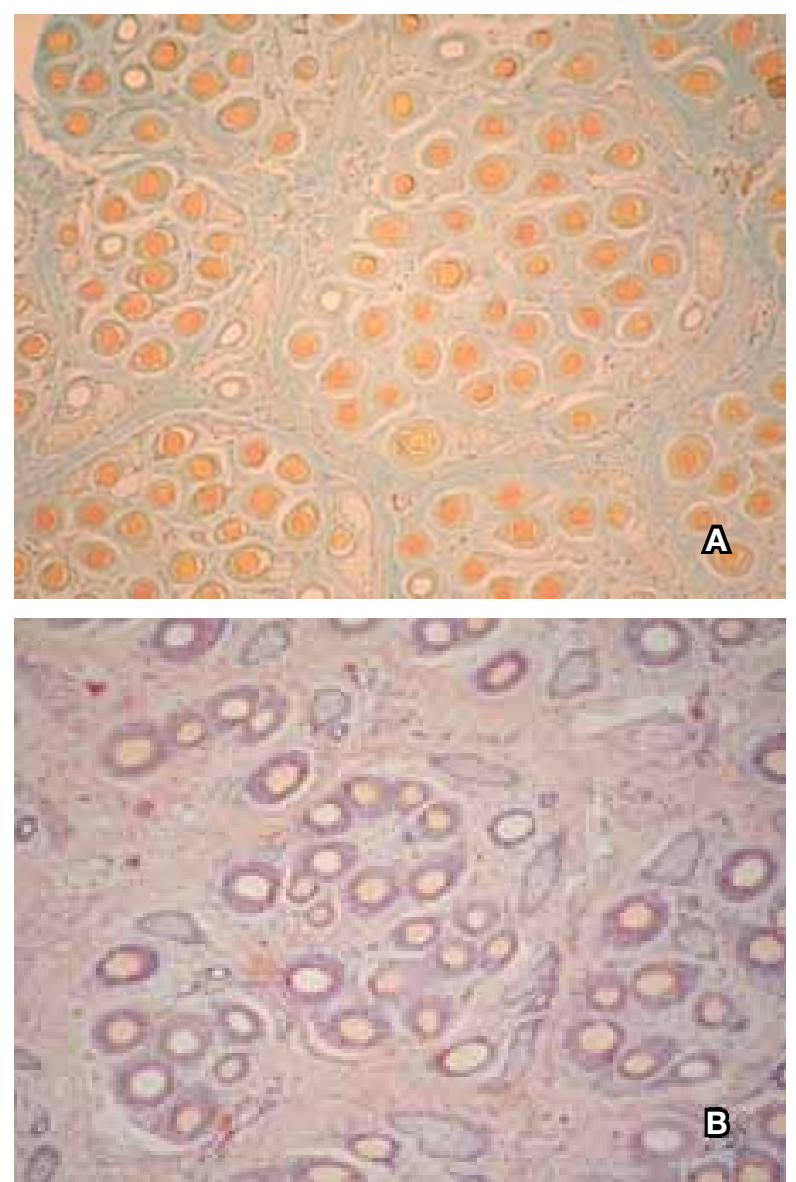

Figura 2. Corte histológico de piel de borrega Ideal, mostrando folículos primarios y secundarios con diferentes coloraciones. A: Sapicsatin, B: HyE, 10X 
tre el segundo y tercer parto, con buen estado sanitario y características fenotípicas homogéneas. Las borregas fueron contemporáneas, producto del cruzamiento de ovejas Ideal con carneros Ideal (borregas Ideal) y con carneros MPM (borregas F1). El diseño experimental fue completamente al azar. Las estadísticas descriptivas incluyeron la media aritmética $(\bar{x})$ el desvío estándar (DE). Los datos se analizaron por ANOVA y las medias se compararon por el test $\mathrm{T}$ utilizando el software Infostat $2010{ }^{17}$, con significancia de $5 \%$.

\section{RESULTADOS Y DISCUSIÓN}

Al analizar los datos del diámetro de fibra se pudo observar que en el grupo de borregas Ideal puras un $50 \%$ presentó diámetros entre 19 y $22,25 \mu \mathrm{m}$, mientras que el otro $50 \%$ se ubicó entre 22,25 y $25,50 \mu \mathrm{m}$. Por su parte, el $63 \%$ del grupo de borregas cruza MPM presentó diámetros entre 19 y $20,31 \mu \mathrm{m}$, mientras que el $37 \%$ restante se ubicó entre 20,31 y $24,10 \mu \mathrm{m}$. Esta distribución demostró que la lana reveló una tendencia a afinarse como efecto del cruzamiento, lo cual constituye una mejoría de este parámetro para la majada.

Los valores consignados ut supra coinciden con los obtenidos en un trabajo similar con la raza Corriedale, donde la distribución para borregas puras mostró un $70 \%$ entre 25 y $28 \mu \mathrm{m}$ de diámetro de fibra, mientras que sus contemporáneas F1 Corriedale x MPM obtuvieron un $60 \%$ por debajo de $22 \mu \mathrm{m}$ de diámetro ${ }^{12}$.

En la Tabla 1 se presentan los resultados correspondientes a las características productivas obtenidas en las borregas Ideal y en las F1. El valor promedio del diámetro de fibra en ovejas madres de la majada Ideal fue $24,3 \mu \mathrm{m}$, en tanto que las borregas Ideal registraron $22,24 \mu \mathrm{m}$ y las borregas F1 Ideal x MPM promediaron $21,06 \mu \mathrm{m}$, valores que resultaron estadísticamente significativos $(\mathrm{p}>0,05)$.

Los resultados de diámetro de fibra en la majada original concuerdan con datos que señalan para la raza Ideal valores entre 22,1 y $26,8 \mu \mathrm{m}^{19,20,30}$. Para MPM no se encontraron publicaciones que hagan referencia al diámetro, solo se mencionan datos citados bajo la anterior denominación de Australian Meat Merino, destacándose como raza productora de lana de elite de menos de $20 \mu \mathrm{m}^{10}$.

Si bien cada raza tiene un rango promedio de diámetro de fibra, hay factores que influyen en este parámetro, tales como edad, sexo, gestación y nivel nutricional. La comparación realizada en el presente trabajo en iguales condiciones de alimentación, sanidad, sexo y edad, permiten atribuir la disminución del diámetro de fibra al cruzamiento con MPM. El diámetro de la lana es una característica de alta heredabilidad, de manera que se pueden conseguir con rapidez cambios en sus valores ${ }^{8,14}$.

Los resultados obtenidos en el cruzamiento utilizando madres Ideal mostraron menor avance en la reducción del diámetro de fibra, que los observados en el cruzamiento con ovejas Corriedale ${ }^{12}$. Sin embargo, coinciden con los reportados por algunos autores que expresan que es normal esperar menores reducciones del diámetro de fibra cuando se parte de una majada con micronaje inferior, es decir que el avance genético sería más gradual en estos casos ${ }^{14}$.

En Australia la reducción del diámetro de fibra de 21,5 a $19 \mu \mathrm{m}$ demandó varios años. En establecimientos de Santa Cruz, Argentina, se citaron disminuciones de 24,5 a $22 \mu \mathrm{m}$ y de 21 a $19,5 \mu \mathrm{m}$ utilizando MPM sobre majadas Ideal ${ }^{28}$. En el INTA Mercedes (Corrientes) durante 5 años se ensayaron dos alternativas tendientes a lograr menos de $22 \mu \mathrm{m}$, una fue la selección por finura de animales de la raza Ideal -de doble propósito- y otra el cruzamiento por absorción de ovejas Ideal con carneros Merino ${ }^{18}$. En la segunda generación, los animales $3 / 4$ Merino produjeron lana de $22,40 \mu \mathrm{m}$, con un coeficiente de variación del diámetro de $18,45 \%$ y $4,09 \%$ de fibras mayores a $30 \mu \mathrm{m}$. Los animales Ideal alcanzaron $23,35 \mu \mathrm{m}, 19,65 \%$ y $6,01 \%$ respectivamente, y no se registraron diferencias en peso vivo de las ovejas, peso de vellón limpio y longitud de la mecha.

En la mesopotamia argentina los indicadores productivos son bajos, con predominancia de lanas entre 25 a $32 \mu \mathrm{m}$ de diámetro ${ }^{13}$, marcadamente más gruesas que las producidas por las borregas de este trabajo. En 2009, alrededor de $15.000 \mathrm{~kg}$ de lana de oveja fueron acopiadas en Goya, Esquina, Perugorría, Curuzú Cuatiá y Sauce, comercializándose por valores que superaron los $\$ 161.000^{6}$. La lana procedente de Curuzú Cuatiá fue tipo "ideal" de $24,5 \mu \mathrm{m}$, en tanto que en otro lote fue de $27,7 \mu \mathrm{m}$, con más del $70 \%$ de rendimiento al lavado. El producto procedente de Sauce y Esquina, obtuvo valores de $28,8 \mu \mathrm{m}$, en tanto que la lana proveniente de Perugorría registró $28,1 \mu \mathrm{m}$ y la de Goya $28,7 \mu \mathrm{m}$ con rinde de $64 \%$ al lavado. Desde el INTA se sostiene que en las regiones subtropicales, con regímenes de precipitaciones de 1100-1300 mm anuales y altas temperaturas, es posible producir lanas más finas utilizando la genética Merino ${ }^{18}$.

Estudios genéticos establecieron que muchos caracteres de piel y vellón pueden ser modificados usando apropiados procedimientos de selección ${ }^{14}$. Sin embargo, la selección de un carácter simple induce además de la respuesta deseada, la modificación de otros no seleccionados. Así, los cambios en el diámetro de fibra pueden ser acompañados por respuestas inversas en la densidad folicular. El tejido folicular que se forma en la piel fetal está bajo el control de poblaciones celulares que son incorporadas a la papila dérmica. El número de células precursoras o pre-papilares que se diferencian en la vida fetal, define la cantidad de tejido folicular que se puede desarrollar y la capacidad innata de producir fibras. La distribución de las células precursoras determina las características individuales que definen su capacidad de producir un tipo particular de fibra. Es decir que los genes manipulan el peso del vellón y la calidad de fibra, por cambios en el número y distribución de las células pre-papilares. Existe correlación positiva del diámetro de fibra con el peso de vellón, rendi- 
miento y longitud de mecha, por lo que al disminuir el grosor de la lana, disminuye el peso del vellón, la longitud de la mecha y el rendimiento ${ }^{26}$.

La longitud de mecha es una de las características más importantes de la lana sucia. Las fibras tienen velocidades de crecimiento que dependen de la época del año y su velocidad individual contribuye al promedio de longitud de mecha al cabo de un año. En el presente trabajo, los resultados no mostraron diferencias significativas (Tabla 1). La longitud de mecha fue $128 \pm 13,5 \mathrm{~mm}$ en borregas Ideal y $137 \pm 19,2$ mm en F1 (Ideal x MPM). Comparado con el ensayo en Corriedale ${ }^{12}$, el cruzamiento con ovejas Ideal produjo vellones con fibras más cortas. Esto es compatible con la estrecha relación que existe con el diámetro: las fibras más finas crecen con mayor lentitud que las más gruesas. En todas las razas, con excepción de Merino Australiano, se encontró que a mayor longitud corresponde un mayor grosor de la fibra ${ }^{15}$.

Según la clasificación usada para separar fardos, las mechas de los dos grupos están en la categoría de excelentes ya que superan los $85 \mathrm{~mm}$. No se encontraron datos que describan en detalle la progresión de la mejoría conseguida con los cruzamientos con MPM, hasta llegar a la longitud de mecha divulgado por los promotores de la raza, que aseguran que se necesitan dos esquilas anuales para aprovechar la longitud de fibra producida. La dimensión depende exclusivamente de la velocidad de crecimiento producida por los folículos secundarios ${ }^{15}$. Se cree que la longitud está controlada por el número, la forma de agrupamiento y la intensidad de la señal emitida por las células prepapilares en la piel del feto ${ }^{25}$.

El MPM se utiliza como un animal mejorador de la calidad de lana, destacándose el aumento del peso del vellón y el menor diámetro de fibra. En este trabajo el peso del vellón de las borregas fue $3,46 \pm 0,24 \mathrm{~kg}$ en Ideal y $3,30 \pm 0,39 \mathrm{~kg}$ en borregas F1 Ideal x MPM, sin diferencias significativas (Tabla 1).

Numerosos datos muestran que animales con lana más fina pesan menos y producen menos lana ${ }^{26}$, sin embargo, actualmente se cree posible afinar la lana sin perder peso de vellón ${ }^{27}$. Los primeros datos surgieron del análisis de los resultados de la prueba de progenie de padres Merino, que ejecuta el INTA junto a la Asociación de Criadores Merino en Pilcaniyeu, comparando padres con progenie de lana más fina a la primer esquila y padres con progenie de lana más gruesa. Los padres más finos fueron también los de mayor peso de vellón aunque de menor peso corporal. Esto indica que existen reproductores que dejan progenie fina con alto peso de vellón, lo que podría explicar los resultados del presente ensayo.

Los programas de registros para ovinos laneros típicamente incluyen en el objetivo de selección al peso de vellón limpio. El rendimiento al lavado (cociente en- tre el peso de la lana lavada, secada y acondicionada) y el peso de vellón sucio son importantes en la fijación del precio, ya que la materia prima para la industria es la fibra limpia ${ }^{2}$. Como regla general, el rendimiento aumenta con el diámetro de la lana y con la cantidad de lluvia, habiendo sido establecido que por cada micrómetro de aumento en el diámetro se produce un aumento en el rendimiento de $0,5 \%$, aproximadamente ${ }^{22}$. En este trabajo (Tabla 1), el rendimiento al lavado no mostró diferencias entre las borregas Ideal y F1, en coincidencia con lo ocurrido con las otras variables estudiadas, excepto el diámetro.

Los resultados obtenidos en este ensayo fueron los esperados ante la utilización de MPM, ya que entre sus características destacadas figura la disposición de las fibras de lana con mejor alineamiento en la mecha, debido a la mayor densidad de folículos y relación $\mathrm{S} / \mathrm{P}$, lo que evitaría que la suciedad quede retenida. Si bien este parámetro responde a una heredabilidad de $0,30^{3} \mathrm{a}$ $0,50^{27}$, el principal factor que influye sobre esta característica es el ambiente. Algunos trabajos alertan sobre el aumento exagerado en el rendimiento (y por ende una probable disminución en la cantidad de cera) como una respuesta correlacionada a la selección por peso de vellón limpio, ya que podría conducir a una disminución en la protección del vellón contra la penetración del agua y el polvo ${ }^{4,16}$, y posiblemente a una mayor incidencia de podredumbre del vellón ${ }^{29}$.

Las bases biológicas que utilizan el sistema de cría que origina al MPM, se fundamentan en la distribución de las células pre-papilares y en la intensidad de señal para el crecimiento de las fibras ${ }^{23-25}$. La densidad de folículos en estos animales es superior a 85 por $\mathrm{mm}^{2} \mathrm{de}$ piel, y la relación S/P es 40/1.

La capacidad de la piel para seguir formando folículos sugiere que el proceso de iniciación es conducido por un mecanismo morfo-genético que no está limitado por la densidad de los sitios de iniciación, ni por la proximidad de otros folículos ${ }^{5}$. El tamaño de la población de células comprometidas en la formación de folículos define la cantidad total de tejido productor de fibra en la piel. El número de células que se distribuye a cada folículo piloso en la iniciación, determina el tamaño final de los folículos y sus fibras. La formación del folículo cesa cuando la mayoría o la totalidad de las células comprometidas se utilizaron ${ }^{25}$. Sin embargo, es 
Tabla 2. Densidad de folículos y relación folículos secundarios/primarios (S/P) en ovejas y borregas Ideal puras y borregas F1 Ideal x MPM.

\begin{tabular}{lcccc}
\hline parámetro & ovejas Ideal & borregas Ideal & borregas $\mathrm{F} 1$ & $\mathrm{p}$ \\
\hline folículos $/ \mathrm{mm}^{2}$ & $39,00 \pm 2,43$ & $51,59 \pm 2,26$ & & $* *$ \\
folículos $/ \mathrm{mm}^{2}$ & $39,00 \pm 2,43$ & & $58,05 \pm 2,43$ & $* *$ \\
folículos $/ \mathrm{mm}^{2}$ & & $51,59 \pm 2,26$ & $58,05 \pm 2,43$ & $\mathrm{NS}$ \\
\hline relación $\mathrm{S} / \mathrm{P}$ & $12,61 \pm 2,02$ & $11,98 \pm 2,29$ & & $\mathrm{NS}$ \\
relación $\mathrm{S} / \mathrm{P}$ & $12,61 \pm 2,02$ & & $13,70 \pm 2,26$ & $\mathrm{NS}$ \\
relación $\mathrm{S} / \mathrm{P}$ & & $11,98 \pm 2,29$ & $13,70 \pm 2,26$ & $*$ \\
\hline
\end{tabular}

NS, no significativo; $* \mathrm{p}<0,05 ; * * \mathrm{p}<0,01$.

importante analizar la relación entre los folículos adyacentes y la interpretación de éstos como una población. La misma heterogeneidad de la población es común a todo tipo de ovino independiente al vellón que posean.

La medición de la densidad es un proceso lento y costoso, lo que explica que la mayoría de los trabajos encontrados estén centrados en los aspectos productivos y no en los morfológicos de calidad de piel, complicando la comparación de resultados. En este trabajo la densidad de folículos en las madres Ideal fue $39 / \mathrm{mm}^{2}$, en las borregas Ideal fue $51,6 / \mathrm{mm}^{2}$, en tanto que en la F1 Ideal x MPM fue significativamente diferente, con $58 / \mathrm{mm}^{2}$ (Tabla 2).

La densidad total de folículos por $\mathrm{mm}^{2}$ no se vio modificada en las borregas. Para animales puros la densidad fue similar a la citada en la bibliografía, donde se detallan valores de 50,2 $\pm 9,7 / \mathrm{mm}^{2}$ para animales Ideal ${ }^{30}$. El número total de folículos depende de la medida cambiante de superficie de piel, variable según la rugosidad de la misma y el tamaño del cuerpo. Es más representativa la relación $\mathrm{S} / \mathrm{P}$, que proporciona un índice de variación razonablemente confiable en la población de folículos secundarios ${ }^{7}$.

La densidad de folículos en este ensayo (Tabla 2) permite explicar morfológicamente la falta de diferencias significativas halladas en el peso del vellón. Al formarse agrupamientos pequeños de células pre-papilares se forman muchos folículos laneros que producen fibras más finas. La mayor cantidad de fibras producidas por el mayor número de folículos fundamenta la invariabilidad del peso del vellón observado, a pesar del menor diámetro de fibra. La relación S/P no mostró diferencias entre madres e hijas Ideal, en tanto que en la comparación entre borregas contemporáneas se destacan diferencias significativas a favor de las borregas F1. Los valores fueron similares a los observados para borregas Ideal $(12,8 \pm 3,84)^{30}$.

En este trabajo el coeficiente de variación (CV) de fibra fue $17 \pm 2,47 \%$ y $16,28 \pm 1,57 \%$ para Ideal y F1, respectivamente, valores inferiores a los encontrados en borregas Corriedale y F1 MPM x Corriedale (20,43 $\pm 2,27 \%$ y $19,62 \pm 2,47 \%$, respectivamente) ${ }^{12}$. En ambos trabajos la diferencia no fue significativa. Puede asumirse que la menor variación cuando se trabajó con Ideal, se debe a que el diámetro de fibra de la raza Ideal presenta menores diferencias. En un trabajo que analizó más de 1000 muestras de vellón, donde el CV de fibras se situó entre 18$19 \%{ }^{1}$, se observó que el parámetro varió fuertemente entre animales (desde 13 a $25 \%$ ), sugiriendo un buen margen para la mejora de la uniformidad de diámetro en muchas majadas. Estadísticamente, el índice W/D no presentó diferencias significativas en ambos ensayos. Este índice marca un avance genético en los parámetros de producción de lana y puede aportar una aproximación muy certera de los cambios producidos en la majada comercial como resultado de un sistema de mejoría ${ }^{11}$.

En conclusión, el cruzamiento MPM con Ideal produjo disminución del diámetro de fibra, con mayor relación $\mathrm{S} / \mathrm{P}$, sin afectar otros parámetros productivos. Este trabajo constituye la primera información local para un mejor entendimiento de los parámetros biológicos que determinan la producción de lanas en el nordeste argentino.

\section{REFERENCIAS}

1. Baxter B, Cotlle J. 1997. Fiber diameter distribution characteristics of midside (fleece) samples and their use in sheep breeding. Internat Wool Textile Org Boston Meeting, report \# 12, 8 p.

2. Cardellino R, Florín A, Rodríguez A. 1977. Determinaciones de la repetibilidad. Boletín Técnico SUL - Secretariado Uruguayo de la Lana 1: 49-52.

3. Cardellino R. 1994. Importancia de las características de la lana. Boletín Técnico SUL - Secretariado Uruguayo de la Lana 10: 43-54.

4. Charlesworth D. 1970. A study of dust contamination in Merino wool in low rainfall areas, Publ. School of Wool and Pastoral Sciences, University of New South Wales, Sydney (Australia), 2052 p.

5. Claxton J. 1964. The determination of patterns with special reference to that of the central primary skin follicles in sheep. J Theor Biol 6: 291-206.

6. Comisión Mixta Provincial del Ovino, Provincia de Corrientes (Argentina). 2004. Diagnóstico de la producción ovina de Corrientes. Rev IDIA XXI 7: 27-33.

7. Corbett JL. 1979. Variation in wool growth with physiological state. In: Physiological and environmental limitation to wool growth (Black JL, Reis PJ, Ed.). University New England Publishing Unit, Armindale (Australia), p. 79.

8. Ensminger M. 1973. Producción ovina, El Ateneo, Buenos Aires, p. 348-378.

9. Federación Lanera Argentina-FLA. 2012. Estadísticas laneras zafra 2011/12 completa. Disponible en www.flasite.com/ftp/anual.pdf. Acceso: enero 2012.

10. Ferguson K. 1995. The evidence for selecting sheep. Austr Farm J (Canberra) 4: 28-31.

11. Ferguson K, Watts J. 1998. The response to selection in the Merino. Proceeding 6th World Congress on Genetics 
Applied to Livestock Production Held in Armidale, Lindsay (Australia), p. 3-10.

12. Flores C, Yáñez E, Carlino M, Bangher G. 2012. Morfología de la piel y producción de lana en cruzamiento absorbente con Merino Multipropósito. Int J Morphol 30: 1434-1441.

13. Gambetta R, Pueyo J. 2004. Producción ovina en la Mesopotamia Argentina. Rev IDIA XXI 7: 3.

14. García G. 1980. Mejoramiento genético de ovinos. Public Docente Facultad de Agronomía, Universidad de Chile 6: 70.

15. García G. 1986. Producción ovina. Ed. Universidad de Chile, Campus Antumapu, 344 p.

16. Hayman R. 1953. Studies in fleece rot of sheep. Aust $J$ Agric Res 4: 430-468.

17. Infostat 2010. Versión 1.1. Grupo Infostat, Facultad de Ciencias Agropecuarias, Universidad Nacional de Córdoba, Argentina.

18. Instituto Nacional de Tecnología Agropecuaria INTA (Argentina). 2009. Intentan producir lanas finas en Mercedes, Corrientes. Disponible en http//www .agro-not.com. ar/art2549.html. Acceso en noviembre 2011.

19. Link P. 1937. Razas ovinas, Casa Peuser, Buenos Aires, $305 \mathrm{p}$.

20. López Magaldi M. 1989. Producción ovina, Ed Albatros, Buenos Aires, $215 \mathrm{p}$.

21. McCloghry C. 1997. Histological technique for the determination of wool follicle density. Wool Technol \& Sheep Breed 45: 2-6.

22. Minola J, Elizondo A. 1989. Praderas y Lanares. Tecnología Ovina Sudamericana, Hemisferio Sur, Buenos Aires, $64 \mathrm{p}$.
23. Moore GP. 1984. Growth and development of follicle populations and critical stages of growth. Proceedings of the Seminar on Wool Production in Western Australia, Perth (Australia), $144 \mathrm{p}$.

24. Moore G, Jackson N, Lax J. 1989. Evidence of a unique developmental mechanism specifying both wool follicle density and fiber size in sheep selected for single skin and fleece characteristics. Genet Res Cambridge 53: 57-62.

25. Moore G, Jackson N, Isaacs K. 1998. Pattern and morphogenesis in skin. J Theor Biol 191: 87-94.

26. Muller J. 1999. Producción de lana súper fina. Anales II Congreso Lanero Argentino, Trelew (Argentina), com. $\mathrm{N}^{\mathrm{o}}$ 333, p. 11.

27. Muller J, Sachero D, Duga L. 2005. Interacción genotipo-ambiente sobre la producción de ovinos de lana superfina en la Patagonia. 2-Calidad de lana. Rev Arg Prod Anim 25: 143-152.

28. Ovis XXI, Consultora sudamericana de producción ovina. 2011. Análisis de la cadena de valor de lanas en la República Argentina. http://www.ovis21.com/ sitio/images/docs/Informe_sobre_cadena_de_valor_2.pdf.

29. Raadsma H, Rogan I. 1987. Genetic variation in resistance to blowfly strike. In: Merino Improvement Programs in Australia (McGuirk BJ Ed), Australian Wool Corp., Melbourne, p. 321-339.

30. Ryder M, Stephenson S. 1968. Wool Growth, Academic Press, London, 805 p. 